\title{
A CLASSIFICATION OF SOME UNIT LORENTZ BALLS AS NON-INTERSECTION BODIES
}

\author{
PATRICK SPENCER
}

(Communicated by Thomas Schlumprecht)

\begin{abstract}
We show that the unit ball of the $n$-dimensional Lorentz space $\ell_{w, q}^{n}$ is not an intersection body for $q>2$ and $n \geq 5$.
\end{abstract}

\section{INTRODUCTION}

A closed, bounded set $K$ in $\mathbb{R}^{n}$ is called a star body if the origin is an interior point of $K$, if for every $x \in K$ each point on the line $[0, x)$ is an interior point of $K$, and if the boundary of $K$ is continuous in the sense that the Minkowski functional

$$
\|x\|_{K}=\min \{a \geq 0: x \in a K\}
$$

is continuous. Following Lutwak 14 we say an origin-symmetric star body $K$ is called an intersection body of a star body $L$ if the radius of $K$ in every direction $\xi \in S^{n}$ is equal to the $(n-1)$-dimensional volume of the intersection of $L$ and the $(n-1)$-dimensional central hyperplane $\xi^{\perp}$ orthogonal to $\xi$. In other words,

$$
\rho_{K}(\xi)=\operatorname{Vol}_{n-1}\left(L \cap \xi^{\perp}\right),
$$

for all $\xi \in S^{n-1}$, where $\rho_{K}(\xi)=\|\xi\|_{K}^{-1}$ is the radial function of $K$. Since we can express $\operatorname{Vol}_{n-1}\left(L \cap \xi^{\perp}\right)$ as

$$
\frac{1}{n-1} \int_{S^{n-1}}\|x\|_{L}^{-n+1} d x=R\left((n-1)^{-1}\|x\|_{L}^{-n+1}\right),
$$

where

$$
\xi \mapsto R f(\xi)=\int_{S^{n-1} \cap \xi^{\perp}} f(x) d x, \quad \xi \in S^{n-1},
$$

denotes the spherical Radon transform of the function $f$, then we can generalize our definition of an intersection body, as in [8], as follows: An origin-symmetric star body $K$ is an intersection body if there exists a finite Borel measure $\mu$ on $S^{n-1}$ such that $\|\cdot\|_{K}^{-1}=R \mu$ in the sense of linear functionals on the space $C\left(S^{n-1}\right)$ of continuous function on the sphere, i.e.

$$
\int_{S^{n-1}}\|x\|_{K}^{-1} f(x) d x=\int_{S^{n-1}} R(f)(x) d \mu
$$

for all $f \in C\left(S^{n-1}\right)$.

Received by the editors December 2, 2011 and, in revised form, April 5, 2012 and April 17, 2012 .

2010 Mathematics Subject Classification. Primary 52A20; Secondary 42A38, 42A82, 46F12.

Key words and phrases. Lorentz space, intersection bodies.

The author would like to thank Alexander Koldobsky for all his guidance and support throughout this paper. 
The concept of an intersection body is important in the dual Brunn-Minkowski theory, introduced by Lutwak in [14. In particular, intersection bodies played an important role in the solution to the Busemann-Petty problem posed in 1956, which can be stated as follows: Suppose $K$ and $L$ are two origin-symmetric convex bodies in $\mathbb{R}^{n}$. If $\operatorname{Vol}_{n-1}\left(K \cap \xi^{\perp}\right) \leq \operatorname{Vol}_{n-1}\left(L \cap \xi^{\perp}\right)$, for all $\xi \in S^{n-1}$, does it follow that $\operatorname{Vol}_{n}(K) \leq \operatorname{Vol}_{n}(L)$ ? The solution was completed in the late 1990's as a result of a series of papers [13, [1, [7, [2, [14, [15], [3, [5, [17, [19, [18, and [6]; see [4. pg. 343] or [12, pg. 3] for a history of the problem and its solution.

By a result of Lutwak in [14, if $K$ is an intersection body, the answer to the question of the Busemann-Petty problem is affirmative for any origin-symmetric star body $L$. On the other hand, if $L$ is a non-intersection body it can be perturbed to construct a body $K$ giving, together with $L$, a counterexample to the BusemannPetty problem. As shown in [5], [18] and [6], every origin-symmetric, convex body in dimension $n \leq 4$ is an intersection body. On the other hand, in dimensions 5 and higher there exist symmetric, convex, non-intersection bodies, each of which provides a counterexample to the Busemann-Petty problem in these dimensions. The first example of a non-intersection body was given by Gardner [3] as a certain rounded cylinder in $\mathbb{R}^{5}$. Other examples of non-intersection bodies in dimensions $n \geq 5$ were given in [19], 6], [9, [11, and [16]. In particular, the unit balls of the spaces $\ell_{q}^{n}$ where $n \geq 5, q>2$ are not intersection bodies, as was proved in [9]. [11] (or [12, sec. 4.4]) provides a characterization of intersection bodies in terms of the second derivative of the norm. For details and more results on intersection bodies see 4 or [12, ch. 4]. In this paper, we show that the unit ball of the $n$-dimensional Lorentz space $\ell_{w, q}^{n}$ is not an intersection body for $q>2$ and $n \geq 5$. Our proof is based on a modification of the second derivative test for intersection bodies found in [11] or [12, pg. 83]. This second derivative test is applicable only when $q>2$, so it should be noted that, as of the writing of this paper, it is unknown to the author whether the unit ball of $\ell_{w, q}^{n}$ is an intersection body for $0<q \leq 2$ and $n \geq 5$.

\section{MAIN THEOREM}

Let $w=\left(a_{1}, \ldots, a_{n}\right) \in \mathbb{R}^{n}$ be such that $a_{1} \geq a_{2} \geq \ldots \geq a_{n} \geq 0$ and define a norm $\|\cdot\|_{w, q}$ on $\mathbb{R}^{n}$ as

$$
\|x\|_{w, q}=\left(a_{1}\left(x_{1}^{*}\right)^{q}+\ldots+a_{n}\left(x_{n}^{*}\right)^{q}\right)^{1 / q},
$$

where $x_{1}^{*}, \ldots x_{n}^{*}$ is the non-increasing rearrangement of $\left|x_{1}\right|, \ldots,\left|x_{n}\right|$. We call

$$
\ell_{w, q}^{n}=\left(\mathbb{R}^{n},\|\cdot\|_{w, q}\right)
$$

the $n$-dimensional Lorentz space.

Now we appeal to the theory of distributions, which one can read more about in [12, sec. 2.5]. Let $\mathbb{N}=\{1,2,3, \ldots\}$ and

$$
\mathbb{N}_{0}^{m}=\left\{\left(a_{1}, \ldots, a_{m}\right): a_{i} \in \mathbb{N} \cup\{0\}, 1 \leq i \leq m\right\} .
$$

Let $\mathscr{S}$ denote the set of all Schwartz functions on $\mathbb{R}^{n}$, i.e.

$$
\mathscr{S}\left(\mathbb{R}^{n}\right)=\left\{\phi \in C^{\infty}\left(\mathbb{R}^{n}\right): \sup _{x \in \mathbb{R}^{n}}\left|x^{\alpha} \partial^{\beta} \phi(x)\right|_{2}<\infty, \forall \alpha, \beta \in \mathbb{N}_{0}^{n}\right\} .
$$


If $f$ is a locally integrable function on $\mathbb{R}^{n}$ and $\phi \in \mathscr{S}\left(\mathbb{R}^{n}\right)$, then we let $\langle f, \phi\rangle$ denote the action of $f$ on $\phi$ when $f$ is viewed as a distribution, i.e.

$$
\langle f, \phi\rangle=\int_{\mathbb{R}^{n}} f(x) \phi(x) d x .
$$

For $\phi \in \mathscr{S}$ we let $\widehat{\phi}(\xi)$ denote the Fourier transform

$$
\int_{\mathbb{R}^{n}} \phi(x) e^{-i x \cdot \xi} d x
$$

of $\phi$ at $\xi$. Here $x \cdot \xi$ denotes the scaler product of $x$ and $\xi$.

Koldobsky [10, Thm. 1], 12, Thm. 4.1] showed that an origin-symmetric star body $K$ is an intersection body if and only if $\|\cdot\|_{K}^{-1}$ is a positive definite distribution; i.e. $\left\langle\|\cdot\|_{K}^{-1}, \widehat{\phi}\right\rangle \geq 0$ for every non-negative $\phi \in \mathscr{S}$. This brings us to the main theorem:

Theorem 2.1. Let $n \geq 5$ and $q>2$. The function $\|\cdot\|_{w, q}^{-p}$ represents a positive definite distribution if and only if $p \in[n-3, n)$.

Let $B_{w, q}^{n}$ denote the unit ball of $\ell_{w, q}^{n}$. We can use Theorem 2.1 along with the fact that $\|x\|_{w, q}=\|x\|_{B_{w, q}^{n}}$ to get the following:

Corollary 2.2. The unit ball of $\ell_{w, q}^{n}$ is not an intersection body for $q>2$ and $n \geq 5$.

The proof of this theorem follows the second derivative test found in [1] or [12, Thm. 4.19], and as such requires Lemma 4.1 below, which is itself a modification of Lemma 1 of [11] (or [12, Lemma 4.20]). The original proof of this lemma relies on the fact that the function $x_{1} \rightarrow\|x\|_{w, q}$ has a continuous first and second derivative on $\mathbb{R}$ for a fixed $\left(x_{2}, \ldots, x_{n}\right) \in \mathbb{R}^{n-1} \backslash\{0\}$. The Lorentz norm doesn't have this property on all of $\mathbb{R}^{n}$, but fortunately the set on which this fails has measure zero.

\section{Properties of the Lorentz nORM}

Fix $q>2$ and $w$ as above. If $x=\left(x_{1}, x_{2}, \ldots, x_{n}\right) \in \mathbb{R}^{n}$, then $y$ will denote $\left(x_{2}, \ldots, x_{n}\right) \in \mathbb{R}^{n-1}$. Let $e_{1}, e_{2}, \ldots, e_{n}$ be an orthonormal basis of $\mathbb{R}^{n}$. Throughout the paper $\|x\|$ denotes $\left\|x_{1} e_{1}+\ldots+x_{n} e_{n}\right\|_{w, q}$ and $\|y\|$ denotes $\left\|e_{2} x_{2}+\ldots+e_{n} x_{n}\right\|$. We will sometimes revert back to $\|x\|_{w, q}$ for emphasis. We let $|\cdot|_{2}$ and $B_{2}^{n}$ denote the norm and unit ball of $\ell_{2}^{n}$ respectively.

Let $S_{n}$ be the group of permutations on $n$ symbols and let

$$
A_{\sigma}=\left\{x \in \mathbb{R}^{n}:\left|x_{\sigma(1)}\right|>\left|x_{\sigma(2)}\right|>\ldots>\left|x_{\sigma(n)}\right|\right\}
$$

for all $\sigma \in S_{n}$. For each such $\sigma$, we note that $A_{\sigma}$ is open and that $\mathbb{R}^{n} \backslash\left(\bigcup_{\sigma} A_{\sigma}\right)$ is of Lebesgue measure zero.

For a given $\sigma \in S_{n}$ and $y=\left(x_{2}, \ldots, x_{n}\right) \in \mathbb{R}^{n-1}$, let $\|x\|_{x_{1}}^{\prime}(\cdot, y)$ and $\|x\|_{x_{1}^{2}}^{\prime \prime}(\cdot, y)$ denote the first and second derivative of the function

$$
x_{1} \rightarrow\left\|x_{1} e_{1}+x_{2} e_{2}+\ldots+x_{n} e_{n}\right\|
$$

respectively and let $A_{\sigma}^{1}(y)=\left\{\xi \in \mathbb{R}:(\xi, y) \in A_{\sigma}\right\}$.

Proposition 3.1. For $q>2, \sigma \in S_{n}$ and a fixed $y=\left(x_{2}, \ldots, x_{n}\right) \in \mathbb{R}^{n-1}$ with $y \neq 0$, the function $x_{1} \rightarrow\left\|x_{1} e_{1}+x_{2} e_{2}+\ldots+x_{n} e_{n}\right\|$ has a continuous first and second derivative on the set $A_{\sigma}^{1}(y)$. 
Moreover, for every $\left(x_{1}, y\right) \in A_{\sigma}$ with $\|y\|=1$ we have

$$
\|x\|_{x_{1}}^{\prime}(0, y)=\|x\|_{x_{1}^{2}}^{\prime \prime}(0, y)=0
$$

and there exists a $K>0$ such that $\|x\|_{x_{1}^{2}}^{\prime \prime}\left(x_{1}, y\right)<K$ for every $y \in \mathbb{R}^{n-1}$ with $\|y\|=1$ and every $x_{1} \in A_{\sigma}^{1}(y)$.

Proof. Fix $\sigma \in S_{n}$ and $y \in \mathbb{R}^{n-1} \backslash\{0\}$. We will now consider the function $x_{1} \rightarrow$ $\left\|x_{1} e_{1}+x_{2} e_{2}+\ldots+x_{n} e_{n}\right\|$ defined on the set $A_{\sigma}^{1}(y)$. This function is even, so it suffices to assume $x_{1} \geq 0$, which implies $\left(\left|x_{1}\right|^{q}\right)^{\prime}=q x_{1}^{q-1}$. Let $i \in\{1, \ldots, n\}$ be such that $x_{i}^{*}=\left|x_{1}\right|$. Since we are only considering $\left(x_{1}, y\right) \in A_{\sigma}$, the index $i$ is constant. Let $C_{\sigma, y}=\sum_{j \neq i} a_{j}\left(x_{j}^{*}\right)^{q}$, which is constant for a fixed $\sigma$ and $y$. A direct calculation shows

$$
\|x\|_{x_{1}}^{\prime}\left(x_{1}, y\right)=\frac{a_{i} x_{1}^{q-1}}{\left(a_{i} x_{1}^{q}+C_{\sigma, y}\right)^{1-\frac{1}{q}}}
$$

and

$$
\|x\|_{x_{1}^{2}}^{\prime \prime}\left(x_{1}, y\right)=\frac{(q-1) a_{i} x_{1}^{q-2} C_{\sigma, y}}{\left(a_{i} x_{1}^{q}+C_{\sigma, y}\right)^{2-\frac{1}{q}}} .
$$

Since $y \neq 0,\|x\|_{x_{1}}^{\prime}$ and $\|x\|_{x_{1}^{2}}^{\prime \prime}$ are both well defined, continuous functions on $A_{\sigma}^{1}(y)$. Notice also that

$$
\|x\|_{x_{1}}^{\prime}(0, y)=\|x\|_{x_{1}^{2}}^{\prime \prime}(0, y)=0 .
$$

Now we would like to find the constant $K$. If $x_{1} \leq C_{\sigma, y}$, then $x_{1}^{q-2} \leq C_{\sigma, y} \leq$ $a_{i} x_{1}^{q}+C_{\sigma, y} \leq\left(a_{i} x_{1}^{q}+C_{\sigma, y}\right)^{2-\frac{1}{q}}$, and hence

$$
\|x\|_{x_{1}^{2}}^{\prime \prime}\left(x_{1}, y\right) \leq(q-1) a_{i} C_{\sigma, y} .
$$

If $C_{\sigma, y}<x_{1}$, then $x_{1}^{-2}<C_{\sigma, y}$, and hence $x_{1}^{q-2} \leq x_{1}^{q} C_{\sigma, y}$. This implies $a_{1} x_{1}^{q-2} \leq$ $a_{1} x_{1}^{q} C_{\sigma, y} \leq a_{1} x_{1}^{q} C_{\sigma, y}+C_{\sigma, y} \leq C_{\sigma, y}^{2-\frac{1}{q}}\left(a_{1} x_{1}^{q}+1\right)^{2-\frac{1}{q}}$. Therefore

$$
\|x\|_{x_{1}^{2}}^{\prime \prime}\left(x_{1}, y\right) \leq(q-1) C_{\sigma, y}^{2-\frac{1}{q}} .
$$

We can assume that $C_{\sigma, y}>1$, so in either case we have bounded $\|x\|_{x_{1}^{2}}^{\prime \prime}$ by a constant which depends on $C_{\sigma, y}^{2}$. We want this constant to be the same for any $y \in \mathbb{R}^{n-1}$ with $\|y\|=1$. In order to do this first notice $B_{w, q}^{n}$ is compact so there exist constants $k_{1}, k_{2}>0$ such that $k_{1} B_{2}^{n} \subset B_{w, q}^{n} \subset k_{2} B_{2}^{n}$, which implies $k_{1}|y|_{2} \leq\|y\| \leq k_{2}|y|_{2}$ for all $y \in \mathbb{R}^{n-1}$. If $M=\max \left(x_{j}: j \neq i\right)$ and $\|y\|=1$, then, for some $c>0$,

$$
\|x\|_{x_{1}^{2}}^{\prime \prime}\left(x_{1}, y\right) \leq\left(c \cdot C_{\sigma, y}\right)^{2} \leq\left(c M k_{2}\right)^{2} .
$$

This finally shows $\|x\|_{x_{1}^{2}}^{\prime \prime}\left(x_{1}, y\right)$ is bounded by a constant independent of $x_{1}$ and $y$, with $\|y\|=1$, and we call this constant $K$.

Proposition 3.1 shows that the functions $\|x\|_{x_{1}}^{\prime}(\cdot, y)$ and $\|x\|_{x_{1}^{2}}^{\prime \prime}(\cdot, y)$ exist on $A_{\sigma}^{1}(y)$ for every fixed $\sigma \in S_{n}$ and every $y \in \mathbb{R}^{n-1} \backslash\{0\}$, which is why we defined the sets $A_{\sigma}$ as we did. 
Proposition 3.2. Let $0<p<n-3$ and fix $\sigma \in S_{n}$.

(i) The function $y \mapsto\|y\|^{-p-2}$ is locally integrable on $\mathbb{R}^{n-1}$.

(ii) For every fixed $y \in \mathbb{R}^{n-1}$, the function $x_{1} \mapsto\left\|x_{1} e_{1}+\ldots+x_{n} e_{n}\right\|$ is a convex differentiable function whose derivative at $x_{1}=0$ is zero, and therefore $\|x\| \geq\|y\|$ for every $x=\left(x_{1}, y\right) \in A_{\sigma}$.

(iii) The function $\|x\|_{x_{1}^{2}}^{\prime \prime}$ is non-negative, homogeneous of degree -1 . If $K$ is the upper bound found in Proposition 13.1, then for every $\left(x_{1}, y\right) \in A_{\sigma}$ with $y \neq 0$ we have

$$
\|x\|_{x_{1}^{2}}^{\prime \prime}(x)=\|x\|_{x_{1}^{2}}^{\prime \prime}\left(\|y\| \frac{x}{\|y\|}\right)=\frac{1}{\|y\|}\|x\|_{x_{1}^{2}}^{\prime \prime}\left(\frac{x}{\|y\|}\right) \leq \frac{K}{\|y\|} .
$$

(iv) Convergence in the limit $\lim _{x_{1} \rightarrow 0}\|x\|_{x_{1}^{2}}^{\prime \prime}\left(x_{1}, y\right)=0$ is uniform with respect to $y \in \mathbb{R}^{n-1}$ when $\|y\|=1$.

Proof.

(i) Let $B_{w, q}^{n}, k_{1}$, and $k_{2}$ be as in the proof of Proposition 3.1 so that $k_{1}|y|_{2} \leq$ $\|y\| \leq k_{2}|y|_{2}$ for all $y \in \mathbb{R}^{n-1}$. For $\lambda>0$, we let $\lambda B_{2}^{n}$ be the origin centered ball of $\ell_{2}^{n}$ with radius $\lambda$. When we switch to polar coordinates we get

$$
\int_{\lambda B_{2}^{n}}\|y\|^{-p-2} d y \leq 2 \pi k_{1}^{-p-2} \frac{\lambda^{n-3-p}}{n-3-p} .
$$

This shows (i) holds because the last expression in (3.5) is finite.

(ii) A direct computation using the definition of convexity shows that the function $x_{1} \mapsto\left\|x_{1} e_{1}+\ldots+x_{n} e_{n}\right\|$ is convex on the set $A_{\sigma}^{1}(y)$. By Proposition 3.1 we know that $\|x\|_{x_{1}}^{\prime}(0, y)=0$, so, by convexity, $\|x\| \geq\|y\|$.

(iii) The homogeneity of $\|x\|_{x_{1}^{2}}^{\prime \prime}$ follows from the fact that $\|x\|_{x_{1}}^{\prime}$ is homogeneous of degree 0.

(iv) For a fixed $y \in \mathbb{R}^{n-1}$ with $\|y\|=1$ let $i$ and $C_{\sigma, y}$ be as in Proposition 3.1 . If $C_{\sigma, y}=0$, then $\|x\|_{x_{1}^{2}}^{\prime \prime}=0$ by (3.3). Otherwise, if $m=\min \left\{a_{j}: j \neq i\right\}$ and $M=\max \left\{a_{j}: j \neq i\right\}$, then $m, M$, and $|y|_{2}$ are all positive, so, with $k_{1}$ and $k_{2}$ as in part (i), we have

$$
\frac{(q-1) a_{i} x_{1}^{q-2} m}{\left(a_{i} x_{1}^{q-2}+M k_{1}^{-1}\right)^{2-\frac{1}{q}}} \leq \frac{(q-1) a_{i} x_{1}^{q-2} C_{\sigma, y}}{\left(a_{i} x_{1}^{q}+C_{\sigma, y}\right)^{2-\frac{1}{q}}} \leq \frac{(q-1) a_{i} x_{1}^{q-2}}{\left(m k_{2}^{-1}\right)^{1-\frac{1}{q}}} .
$$

The left and right hand sides of (3.6) are independent of $y$, so (iv) holds.

\section{LEMMA}

Define a family of even Schwartz functions $\left(\phi_{m}\right)_{m \in \mathbb{N}}$ as

$$
\phi_{m}(x)=h_{m}\left(x_{1}\right) u\left(x_{2}, \ldots, x_{n}\right), \quad x \in \mathbb{R}^{n},
$$

where for each $m \in \mathbb{N}$,

$$
h_{m}\left(x_{1}\right)=\frac{m}{\sqrt{2 \pi}} \exp \left(-\frac{x_{2}^{2} m^{2}}{2}\right)
$$

and

$$
u\left(x_{2}, \ldots, x_{n}\right)=\frac{1}{(2 \pi)^{(n-1) / 2}} \exp \left(-\frac{x_{2}^{2}+\ldots+x_{n}^{2}}{2}\right) .
$$


Lemma 4.1. Let $p \in(0, n-3)$. For every $\epsilon>0$ there exists $N \in \mathbb{N}$ such that $-\epsilon \leq\left\langle\left(\|\cdot\|^{-p}\right)_{x_{1}}^{\prime \prime}, \phi_{m}\right\rangle$ whenever $m \geq N$.

Proof. We will first show this theorem holds when $\left\langle\left(\|\cdot\|^{-p}\right)_{x_{1}}^{\prime \prime}, \phi_{m}\right\rangle$ is replaced with

$$
\int_{A_{\sigma}}\|x\|^{-p} \frac{d^{2}}{d x_{1}^{2}} \phi_{m}(x) d x
$$

where $\sigma \in S_{n}$ is arbitrary.

Fix $\sigma \in S_{n}$. By Fubini's theorem we have

$$
\int_{A_{\sigma}}\|x\|^{-p} \frac{d^{2}}{d x_{1}^{2}} \phi_{m}(x) d x=\int_{\mathbb{R}^{n-1}} u(y)\left(\int_{A_{\sigma}^{1}(y)}\|x\|^{-p} \frac{d^{2}}{d x_{1}^{2}} h_{m}\left(x_{1}\right) d x_{1}\right) d y .
$$

Since $\left(\|x\|^{-p}\right)_{x_{1}^{2}}^{\prime \prime}(\cdot, y)$ exists on each $A_{\sigma}^{1}(y)$ and since $h_{m}\left(x_{1}\right) \rightarrow 0$ as $x_{1} \rightarrow \pm \infty$ for all $m \in \mathbb{N}$, we can integrate by parts twice to see that (4.2) is equal to

$$
\int_{\mathbb{R}^{n-1}} u(y)\left(\int_{A_{\sigma}^{1}(y)}\left(\|x\|^{-p}\right)_{x_{1}^{2}}^{\prime \prime}\left(x_{1}, y\right) h_{m}\left(x_{1}\right) d x_{1}\right) d y .
$$

Now notice that

$$
\left(\|x\|^{-p}\right)_{x_{1}^{2}}^{\prime \prime}(x)=p(p+1)\|x\|^{-p-2}\left(\|x\|_{x_{1}}^{\prime}(x)\right)^{2}-p\|x\|^{-p-1}\|x\|_{x_{1}^{2}}^{\prime \prime}(x),
$$

so that (4.3) breaks into the sum of two integrals, the first of which is positive and the second is negative. Therefore it suffices to show that the second integral

$$
\int_{\mathbb{R}^{n-1}} u(y)\left(\int_{A_{\sigma}^{1}(y)}\left(\|x\|^{-p-1}\|x\|_{x_{1}^{2}}^{\prime \prime}\left(x_{1}, y\right) h_{m}\left(x_{1}\right) d x_{1}\right) d y\right.
$$

converges to 0 as $m$ grows large.

Let $\epsilon>0$. By part (i) of Lemma [3.2, $\|y\|^{-p-2}$ is locally integrable on $\mathbb{R}^{n-1}$; hence

$$
L=\int_{\mathbb{R}^{n-1}}\|y\|^{-p-2} u(y) d y<\infty .
$$

By (3.5) we can choose $d>0$ small enough so that

$$
\int_{\|y\|<d}\|y\|^{-p-2} u(y) d y<\frac{\epsilon}{3 K}
$$

where $K$ is the constant from Proposition 3.1 By (ii) and (iii) of Lemma 3.2 we have

$$
\begin{aligned}
& \int_{\|y\|<d} u(y)\left(\int_{A_{\sigma}^{1}(y)}\|x\|^{-p-1}\|x\|_{x_{1}^{2}}^{\prime \prime}\left(x_{1}, y\right) h_{m}\left(x_{1}\right) d x_{1}\right) d y \\
& \leq \int_{\|y\|<d} u(y)\left(\int_{A_{\sigma}^{1}(y)}\|y\|^{-p-2} h_{m}\left(x_{1}\right) d x_{1}\right) d y \\
& \leq K \int_{\mathbb{R}} h_{m}\left(x_{1}\right) d x_{1} \int_{\mathbb{R}^{n-1}}\|y\|^{-p-2} u(y) d y \leq \frac{\epsilon}{3} .
\end{aligned}
$$

By (iv) there exists a $\delta>0$ such that

$$
\|x\|_{x_{1}^{2}}^{\prime \prime}\left(x_{1}, y\right)<\frac{\epsilon}{3 L}
$$


whenever $\left|x_{1}\right|<\delta$ and $\|y\|=1$. By (iii) the second derivative of $\|x\|_{x_{1}^{2}}^{\prime \prime}$ is homogeneous of degree -1 , so this, along with uniform convergence, implies that

$$
\|x\|_{x_{1}^{2}}^{\prime \prime}\left(x_{1}, y\right)=\frac{1}{\|y\|}\|x\|_{x_{1}^{2}}^{\prime \prime}\left(\frac{x_{1}}{\|y\|}, \frac{y}{\|y\|}\right) \leq \frac{\epsilon}{3 L\|y\|}
$$

whenever $\left|x_{1}\right| /\|y\|<\delta$.

Now consider the sets

$$
\begin{aligned}
& E_{1}(y):=\left\{x_{1} \in A_{\sigma}^{1}(y):\left|x_{1}\right|<\delta d\right\}, \\
& E_{2}(y):=\left\{x_{1} \in A_{\sigma}^{1}(y):\left|x_{1}\right|>\delta d\right\} .
\end{aligned}
$$

If $\|y\| \geq d$ and $x_{1} \in E_{2}(y)$, then $\left|x_{1}\right| /\|y\|<\delta$, so we can use (4.8) to get

$$
\begin{aligned}
& \int_{\|y\|>d} u(y)\left(\int_{E_{1}(y)}\|x\|^{-p-1}\|x\|_{x_{1}^{2}}^{\prime \prime}\left(x_{1}, y\right) h_{m}\left(x_{1}\right) d x\right) d y \\
& \leq \frac{\epsilon}{3 L} \int_{\mathbb{R}} h_{m}\left(x_{1}\right) d x_{1} \int_{\mathbb{R}^{n-1}}\|y\|^{-p-2} u(y) d y \leq \frac{\epsilon}{3} .
\end{aligned}
$$

Finally, notice for a given $\eta>0$ that

$$
\lim _{m \rightarrow \infty} \int_{\left\{x_{1}:\left|x_{1}\right|>\eta\right\}} h_{m}\left(x_{1}\right) d x_{1}=0
$$

hence we can choose $N \in \mathbb{N}$ so that for all $m>N$, the following holds:

$$
\int_{\left\{x_{1}:\left|x_{1}\right|>\delta d\right\}} h_{m}\left(x_{1}\right) d x_{1}<\frac{\epsilon}{3 K L} .
$$

This along with (ii) and (iii) implies for all $m>N$,

$$
\begin{aligned}
& \int_{\|y\| \geq d} u(y)\left(\int_{E_{2}(y)}\|x\|^{-p-1}\|x\|_{x_{1}^{2}}^{\prime \prime}\left(x_{1}, y\right) h_{m}\left(x_{1}\right) d x\right) d y \\
& \leq K \frac{\epsilon}{3 L} \int_{\mathbb{R}} h_{m}\left(x_{1}\right) d x_{1} \int_{\mathbb{R}^{n-1}}\|y\|^{-p-2} u(y) d y \leq \frac{\epsilon}{3} .
\end{aligned}
$$

Combining (4.6), (4.9), and (4.11) gives us

$$
\lim _{m \rightarrow \infty} \int_{\mathbb{R}^{n-1}} u(y)\left(\int_{A_{\sigma}^{1}(y)}\|x\|^{-p-1}\|x\|_{x_{1}^{2}}^{\prime \prime}\left(x_{1}, y\right) h_{m}\left(x_{1}\right) d x_{1}\right) d y=0 .
$$

Since $\epsilon$ and $\sigma$ were arbitrary we must have that this theorem holds when $\left\langle\left(\|\cdot\|^{-p}\right)_{x_{1}}^{\prime \prime}, \phi_{m}\right\rangle$ is replaced with $\int_{A_{\sigma}}\|x\|^{-p} \frac{d^{2}}{d x_{1}^{2}} \phi_{m}(x) d x$, where $\sigma \in S_{n}$. Notice that

$$
\begin{aligned}
\left\langle\left(\|x\|^{-p}\right)_{x_{1}^{2}}^{\prime \prime}, \phi_{m}\right\rangle & =\int_{\mathbb{R}^{n}}\|x\|^{-p} \frac{d^{2}}{d x_{1}^{2}} \phi_{m}(x) d x \\
& =\sum_{\sigma \in S_{n}} \int_{A_{\sigma}}\|x\|^{-p} \frac{d^{2}}{d x_{1}^{2}} \phi_{m}(x) d x .
\end{aligned}
$$

$S_{n}$ is finite, so (4.13) along with our previous work completes the lemma. 


\section{Proof OF THE MAIN THEOREM}

Proof. Let $n \geq 5$. With $B_{w, q}^{n}$ still denoting the unit ball of $\ell_{w, q}^{n}$ we have that $B_{w, q}^{n}$ is an origin-symmetric convex body in $\mathbb{R}^{n}$, so Corollary 4.9 of [12] shows that if $p \in[n-3, n)$, then $\|x\|_{B_{w, q}^{n}}^{-p}=\|\cdot\|_{w, q}^{-p}$ represents a positive definite distribution.

Now suppose $p \in(0, n-3)$ and that, by way of contradiction, $\|\cdot\|^{-p}=\|\cdot\|_{w, q}^{-p}$ is a positive definite distribution. By Corollary 2.26 of [12] there exists a finite Borel measure $\mu$ on $S^{n-1}$ such that for every even test function $\phi$,

$$
\left\langle\|\cdot\|^{-p}, \phi\right\rangle=\int_{\mathbb{R}^{n}}\|x\|^{-p} \phi(x) d x=\int_{S^{n-1}}\left(\int_{0}^{\infty} t^{p-1} \widehat{\phi}(t \xi) d t\right) d \mu(\xi) .
$$

Since $\left(\partial_{x_{1}}^{2} \phi\right)^{\wedge}(\xi)=-\xi_{1}^{2} \widehat{\phi}(\xi)$ we have

$$
\begin{aligned}
\left\langle\left(\|\cdot\|^{-p}\right)_{x_{1}^{2}}^{\prime \prime}, \phi\right\rangle & =\left\langle\|\cdot\|^{-p},\left(\partial_{x_{1}}^{2} \phi\right)(\xi)\right\rangle \\
& =-\int_{S^{n-1}}\left(\int_{\mathbb{R}}|t|^{p+1} \widehat{\phi}(t \xi) d t\right) \xi_{1}^{2} d \mu(\xi) .
\end{aligned}
$$

This holds for all even test functions $\phi$, so let us consider the functions $\phi_{m}$ as defined in (4.1). Taking the Fourier transform we have

$$
\widehat{\phi}_{m}(\xi)=\exp \left(-\frac{\xi_{1}^{2}}{2 m^{2}}\right) \exp \left(-\frac{\xi_{2}^{2}+\ldots+\xi_{n}^{2}}{2}\right) .
$$

Using (5.1), (5.2), and (5.3) along with the change of variable

$$
x=\frac{t^{2}}{2} \exp \left(\frac{\xi_{1}^{2}}{m^{2}}+\xi_{2}^{2}+\ldots+\xi_{n}^{2}\right)
$$

gives us

$$
\begin{aligned}
\left\langle\left(\|\cdot\|^{-p}\right)_{x_{1}}^{\prime \prime}, \phi_{m}\right\rangle & =-2 \int_{S^{n-1}}\left(\int_{0}^{\infty} t^{p+1} \widehat{\phi_{m}}(t \xi) d t\right) \xi_{1}^{2} d \mu(\xi) \\
& =-2^{(p+1) / 2} \Gamma\left(\frac{p+2}{2}\right) \int_{S^{n-1}} \xi_{1}^{2}\left(\frac{\xi_{1}^{2}}{m^{2}}+\xi_{2}^{2}+\ldots+\xi_{n}^{2}\right) d \mu(\xi) \\
& \leq-2^{(p+1) / 2} \Gamma\left(\frac{p+2}{2}\right) \int_{S^{n-1}} \xi_{1}^{2} d \mu(\xi),
\end{aligned}
$$

where the last inequality follows because $\xi \in S^{n-1}$. By Lemma 4.1, we can make the left side of (5.4) as close to zero as we like by taking $m$ large enough. $\Gamma$ is positive on $(0, \infty)$, so the integral on the right side of (5.4) is non-positive; hence

$$
\int_{S^{n-1}} \xi_{1}^{2} d \mu(\xi)=0
$$

This implies $\mu$ is supported on the intersection of the hyperplane $\xi_{1}=0$ and the sphere $S^{n-1}$. By (5.2) we have $\left(\|x\|^{-p}\right)_{x_{1}}^{\prime \prime}(x)=0$ on each $A_{\sigma}$, which implies for each $\sigma \in S_{n}$ that there exist two functions $g_{1, \sigma}\left(x_{2}, \ldots, x_{n}\right)$ and $g_{1, \sigma}\left(x_{2}, \ldots, x_{n}\right)$ such that $\|x\|^{-p}=g_{1, \sigma}\left(x_{2}, \ldots, x_{n}\right)+x_{1} g_{2, \sigma}\left(x_{2}, \ldots, x_{n}\right)$ on $A_{\sigma}$. This is a contradiction because we know $\|x\|^{-p} \rightarrow 0$ as $x_{1} \rightarrow \infty$ for all fixed $\left(x_{2}, \ldots, x_{n}\right) \in \mathbb{R}^{n} \backslash\{0\}$, but this new representation of $\|x\|^{-p}$ as a linear function of $x_{1}$ shows $\|x\|^{-p} \rightarrow \infty$ on each $A_{\sigma}$ as $x_{1} \rightarrow \infty$. This completes the proof of the main theorem. 


\section{Acknowledgements}

The author would like to thank his advisor, Alexander Koldobsky, for valuable feedback and guidance throughout the research and preparation of this article. He would also like to thank the referee for useful comments which have imporoved this article. Lastly, the author would like to thank the University of Missouri for support during his studies.

\section{REFERENCES}

[1] Keith Ball, Some remarks on the geometry of convex sets, Geometric aspects of functional analysis (1986/87), Lecture Notes in Math., vol. 1317, Springer, Berlin, 1988, pp. 224-231, DOI 10.1007/BFb0081743. MR.950983 (89h:52009)

[2] J. Bourgain, On the Busemann-Petty problem for perturbations of the ball, Geom. Funct. Anal. 1 (1991), no. 1, 1-13, DOI 10.1007/BF01895416. MR.1091609 (92c:52008)

[3] R. J. Gardner, Intersection bodies and the Busemann-Petty problem, Trans. Amer. Math. Soc. 342 (1994), no. 1, 435-445, DOI 10.2307/2154703. MR1201126 (94e:52008)

[4] Richard J. Gardner, Geometric tomography, Encyclopedia of Mathematics and its Applications, vol. 58, Cambridge University Press, Cambridge, 1995. MR1356221 (96j:52006)

[5] R. J. Gardner, A positive answer to the Busemann-Petty problem in three dimensions, Ann. of Math. (2) 140 (1994), no. 2, 435-447, DOI 10.2307/2118606. MR1298719 (95i:52005)

[6] R. J. Gardner, A. Koldobsky, and T. Schlumprecht, An analytic solution to the BusemannPetty problem on sections of convex bodies, Ann. of Math. (2) 149 (1999), no. 2, 691-703, DOI 10.2307/120978. MR:1689343(2001b:52011)

[7] Apostolos A. Giannopoulos, A note on a problem of H. Busemann and C. M. Petty concerning sections of symmetric convex bodies, Mathematika 37 (1990), no. 2, 239-244, DOI 10.1112/S002557930001295X. MR1099772(92c:52009)

[8] P. Goodey, E. Lutwak, and W. Weil, Functional analytic characterizations of classes of convex bodies, Math. Z. 222 (1996), no. 3, 363-381, DOI 10.1007/PL00004539. MR1400197 (97i:52002)

[9] Alexander Koldobsky, Intersection bodies in $R^{4}$, Adv. Math. 136 (1998), no. 1, 1-14, DOI 10.1006/aima.1998.1718. MR.1623669 (99j:52013)

[10] Alexander Koldobsky, Intersection bodies, positive definite distributions, and the BusemannPetty problem, Amer. J. Math. 120 (1998), no. 4, 827-840. MR1637955 (99i:52005)

[11] Alexander Koldobsky, Second derivative test for intersection bodies, Adv. Math. 136 (1998), no. 1, 15-25, DOI 10.1006/aima.1998.1719. MR1623670(99j:52014)

[12] Alexander Koldobsky, Fourier analysis in convex geometry, Mathematical Surveys and Monographs, vol. 116, American Mathematical Society, Providence, RI, 2005. MR2132704 (2006a:42007)

[13] D. G. Larman and C. A. Rogers, The existence of a centrally symmetric convex body with central sections that are unexpectedly small, Mathematika 22 (1975), no. 2, 164-175. MR0390914 (52 \#11737)

[14] Erwin Lutwak, Intersection bodies and dual mixed volumes, Adv. in Math. 71 (1988), no. 2, 232-261, DOI 10.1016/0001-8708(88)90077-1. MR963487(90a:52023)

[15] Michael Papadimitrakis, On the Busemann-Petty problem about convex, centrally symmetric bodies in $\mathbf{R}^{n}$, Mathematika 39 (1992), no. 2, 258-266, DOI 10.1112/S0025579300014996. MR:1203282(94a:52019)

[16] M. Yaskina, Non-intersection bodies, all of whose central sections are intersection bodies, Proc. Amer. Math. Soc. 135 (2007), no. 3, 851-860 (electronic), DOI 10.1090/S0002-993906-08530-3. MR2262882(2007i:52007)

[17] Gao Yong Zhang, Centered bodies and dual mixed volumes, Trans. Amer. Math. Soc. 345 (1994), no. 2, 777-801, DOI 10.2307/2154998. MR.1254193(95d:52008) 
[18] Gao Yong Zhang, A positive solution to the Busemann-Petty problem in $\mathbb{R}^{4}$, Ann. of Math. 149 (1999), 535-543. MR 1689339

[19] Gao Yong Zhang, Intersection bodies and the Busemann-Petty inequalities in $\mathbf{R}^{4}$, journal=Ann. of Math. (2), volume=140, date=1994, number=2, pages=331-346, issn=0003486X, review $=M F 1298716(95 i: 52004)$, doi=10.2307/2118603,.

Department of Mathematics, 202 Mathematical Sciences Building, University of MisSOURi, Columbia, Missouri 65211

E-mail address: patrick.spencer@mail.mizzou.edu 